\title{
Radiation damage in electron cryomicroscopy (cryoEM)
}

\author{
Richard Henderson $^{1}$ \\ ${ }^{1}$ MRC Laboratory Of Molecular Biology, Cambridge, United Kingdom \\ E-mail: rh15@mrc-Imb.cam.ac.uk
}

Radiation damage to biological structures by irradiation with neutrons, electrons or X-rays [1] involves ionization, covalent bond breakage, and release of volatile molecular fragments especially hydrogen as radiation products. Some amino acid side chains are more readily damaged than others. Phenylalanine, tyrosine and tryptophan side chains, as well as nucleotide bases are more radiation resistant than other parts of the protein or nucleic acid. In electron diffraction from 2D or 3D crystals, the most obvious effect is a gradual fading of the diffraction spots that can be roughly characterized by an increase in B-factor with dose. In electron cryomicroscopy (cryoEM), there are two additional effects that compromise the quality of the electron images [2]. First, beam-induced specimen charging due to secondary electron emission can cause electrostatic effects that blur the image. Second, bond breakage and the resulting increase in interatomic distances from covalent to van der Waals bonding causes an increase in internal pressure that is only partly relieved by evaporation of volatile fragments including hydrogen. This internal stress causes beam-induced specimen motion that again blurs the image. In practice, this beam-induced specimen motion and charging manifests itself as a reduction in information content at the beginning of the electron exposure, since this is where charging and internal stresses change most rapidly. Typical exposures in cryoEM involve a total dose of $20-30$ electrons $/ \AA * * 2$, and that part of the image from the first 3-4 electrons/ $\AA * * 2$ has a much lower information content than it should. Since specimens for cryoEM are normally prepared by plunge-freezing into liquid ethane at $-180^{\circ} \mathrm{C}$ using the method developed by Jacques Dubochet in the $1980 \mathrm{~s}$, the macromolecular structures being studied are always embedded in amorphous ice, which is itself subject to radiation damage. Exposure of 1 electron/ $\AA * * 2$ with $300 \mathrm{keV}$ electrons causes water molecules in pure amorphous ice to move by $\sim 1 \AA$ [3]. The illustration below, figure 3 from reference [3], allowed the extent of the motion of the water molecules to be determined. This random motion of the water molecules in the suspending medium also causes pseudo-Brownian motion of the embedded macromolecules, but fortunately this is much smaller than the two major causes of image blurring. We conclude that cryoEM images would be greatly improved if the remaining problems of beam-induced specimen motion and charging could be reduced.

[1] Henderson R. (1995) Quart. Rev. Biophys. 28, 171-193.

[2] Vinothkumar, K.R. \& Henderson R. (2016) Quart. Rev. Biophys. 49, e13, 1-25.

[3] McMullan, G. et al (2015) Ultramicroscopy 158, 26-32.

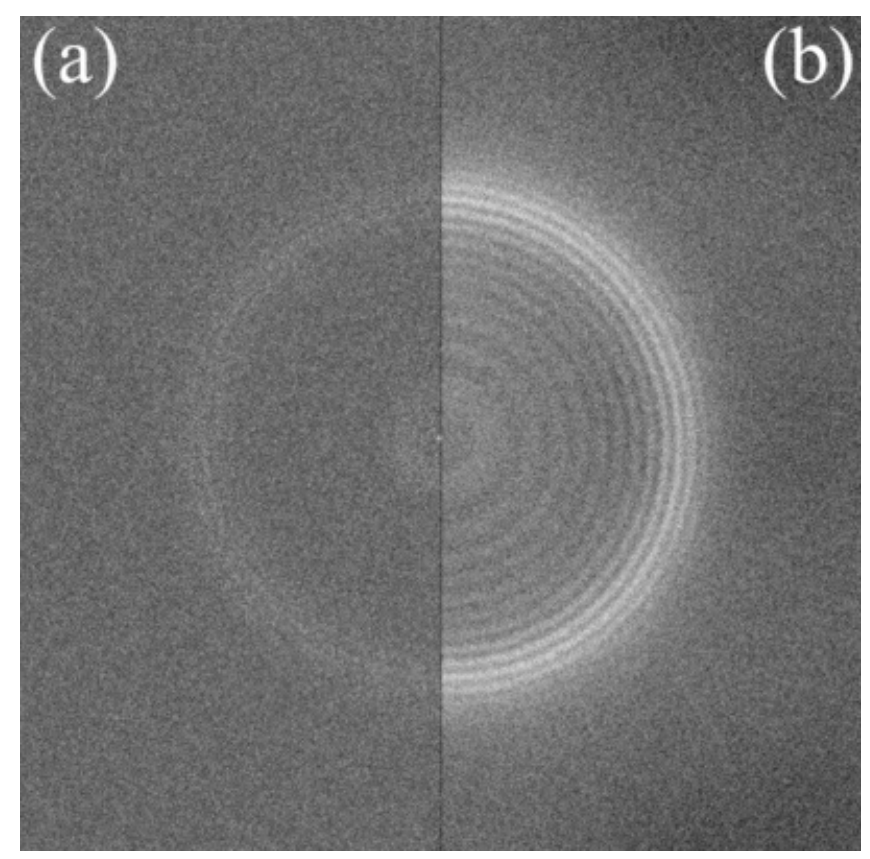

Keywords: B-factor, specimen charging, specimen motion 